DOI: 10.2478/v10122-009-0008-8

\title{
MA'A LEXICON AND AFRO-ASIATIC IV: MA'A $\hat{s}-$
}

\author{
GÁBOR TAKÁCS
}

\begin{abstract}
Gábor Takács. Ma'a Lexicon and Afro-Asiatic IV: Ma'a ŝ-. Lingua Posnaniensis, vol. LI/2009. The Poznań Society for the Advancement of the Arts and Sciences. PL ISSN 0079-4740, ISBN 978-83-7654-080-1, pp. 125-131

The paper represents part of a longer series that examines the lexical stock of the Ma'a language, an exotic Mischsprache combining a Bantu grammar with a basically Cushitic (henceforth, Afro-Asiatic) lexicon, from the standpoint of etymology. This part contains (mostly new) etymologies of Ma'a words with the initial lateral sibilant.

Gábor Takács, Székesfehérvár 8000, Semmelweis u. 19, Hungary, gabtak@mail.datatrans.hu
\end{abstract}

\section{INTRODUCTION}

Ma'a is a Mischsparche in the Usambara mountains, in the northeast of Tanzania. Its non-Cushitic/Afro-Asiatic grammar is basically Bantu. There are strong objections among some specialists against the South Cushitic character of the Ma'a lexicon maintained, e.g., by Ch. Ehret (1980). With respect to the grammatical system of Ma'a, there is a doubt whether Ma'a has ever been an Afro-Asiatic language at all. Nevertheless, the lexical stock clearly reveals not only the South Cushitic, but also a deeper Afro-Asiatic lexical heritage in Ma'a.

This series of papers is devoted to analyzing just this segment of the Ma'a lexicon through regular correspondences, an evidence demonstrating the inherited Afro-Asiatic nature of this (once?) South Cushitic language. The individual parts of the series are usually arranged according to anlaut consonants of Ma'a. The present part consists of the Ma'a lexicon with $\hat{s}^{-}$.

\section{Ma'a $\hat{s}(h l)<\mathrm{SCu} . * \hat{s}<\mathrm{AA} * \hat{s}$}

Contrary to Ma'a, most South Cushitic languages make a distinction between the voiceless lateral sibilant $(* \hat{s})$ and the voiceless lateral affricate $(* \hat{c})$, which have usually merged in most of the Afrasian daughter languages (in nearly all Semitic languages, Egyptian, Berber, Cushito-Omotic). The correspondence of SCu. ${ }^{*} \hat{s}=\mathrm{Sem}$. ${ }^{*} \hat{s}$ was examined by DolgopolsKy (1987, 200-205, \#39-79), who supposed also an additional correspondence of SCu. ${ }^{*} \hat{s}=$ Sem. *h-l \& *h-l, which, however, needs further confirmation. 


\begin{tabular}{|l|l|l|l|l|l|l|l|l|}
\hline $\mathrm{Cu}$. & Rift & Ma'a & Dhl. & Sem. $^{1}$ & Eg. & Ch. & AS & AA \\
\hline$* \hat{s}$ & $* \hat{s} / * h l$ & $\hat{s} / h l$ & $\hat{s} / 4$ & $*_{2}$ & $\check{s}$ & $* \hat{s}$ & $* l$ & $* \hat{s}$ \\
\hline
\end{tabular}

1.1. Ma'a -ŝá "to stab, pierce", hence $m$-ŝa'é "thorn" [Ehr. 1974 MS, 41] | Dhl. $\hat{s} w a h$ "to pinch" [EEN 1989, 26; Tosco 1991, 142] < SCu. * $\hat{s}^{(w)} a h$ - [GT].

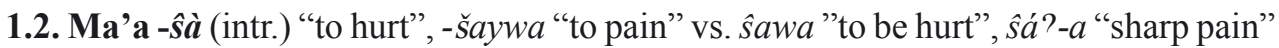
| Irq. $\hat{s} a$ ? h-es- (tr.) "to hurt (so.)", cf. sahah- (intr.) "to hurt", Alg. siha $a$-ame "grief, sorrow" < SCu. *şaha?- "to pain, hurt" [Ehr.] (SCu.: Ehr. 1980, 210, \#20-21) ||| WCh.: Angas-Sura $* l \bar{a}_{2}[\mathrm{reg} .<* \hat{s} a H]$ "to feel pain" [GT 2004, 218] > i.a. Angas lée "1. schmerzen, krank sein, 2. Krankheit" [Jng. 1962 MS, 22] = le e "to be sick, pain" [Hfm.], Sura láa "schmerzen" [Jng. 1963, 72] = laa "to pain" [Hfm.], Goemay laa "to pain, give pain, feel pain" [Srl. 1937, 119] = laa "to pain" [Hfm.] (AS: Hfm. 1975, 21, \#129) < AA * $\hat{s}-h$-(?) "to feel pain" [GT].

1.3. Ma'a ŝahá "to burn", -ŝhahá 'iža "to burn (tr.)" [Ehr.] | Dhl. $\hat{s} a h$ - "to burn (sg.)" [Ehr.] = "to burn up" [EEN 1989, 26] = "to put on fire" [TosCo 1991, 142] (SCu.: Ehr. 1980, 210,\#18).

1.4. Ma'a -ŝaha?é "to scarify, tattoo"| Brg. $\hat{s} a$ ?a-niya "scar" < SCu. *îhaha?- "to leave scar” [GT] (SCu.: Ehret 1980, 209, \#22) ||| WCh. *ŝaH”- [GT]: Hausa ša ššááwà "hereditary tribal marks cut or tattooed on face or body", šáššàùtáá "1. to cut šaššááwà on so., scarify so. in cupping, make small cuts in, 2. lance" [Abr. 1962, 804] | AS *lā $\sim$ *le [reg. < * $\hat{s} a H]$ "ulcer, wound" [GT 2004, 217] > i.a. Sura láa "Wunde" [Jng. 1963, 72] = laa "ulcer" [Krf.], Kofyar léé "scars for decoration of the face and body" [Ntg. 1967, 23] | NBch. *ŝaw"tattoo" [Skn. 1977, 43] < AA * $\hat{s}-h-(?)$ "wound, scar" [GT].

1.5. Ma'a sámu "to be sweet" [Ehr.] | Dhl. sắhame "sweet" [EEN 1989, 26; TosCo 1991, 142] (SCu.: Ehr. 1980, 210, \#19).

1.6. Ma'a *îahw a [GT] > ma-ŝahá "anger", -ŝawa "to be angry" [Ehr. $1974 \mathrm{MS}, 41-42]$

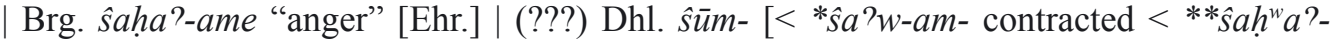
am-?] "to be angry" [EEN 1989, 26] = "to get upset, angry" [Tosco 1991, 142]< SCu. *sahwa?- "to be angry" [GT]?

NB: Alternatively (albeit less probably), we may assume a hypothetic var. Ma'a *sak $k^{w}$ - (cf. the well-attested interchange of Ma'a -k- -h- $\sim-h-<$ SCu. *-k-), cf. Irq. sấa "to fight, argue" [Mgw. 1989, 101]| Dhl. ŝak- "to quarrel, be angry", sàk-a "anger" [Ehr. 1980, 209].

1.7. Ma'a -ŝaka - $\hat{\boldsymbol{s}} \boldsymbol{a} \mathbf{h} \boldsymbol{a}$ "to bear (child, fruit, etc.)" | Irq. $\hat{\boldsymbol{s}} a q^{w_{-}}$"to present, bring into

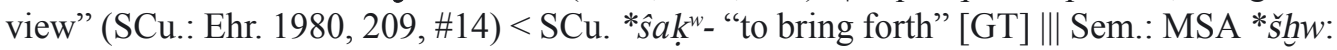
Jbl. šhé "to pull out, extract (as e.g. a book from a case)" [Jns. 1981, 264] $<$ AA * $\hat{s}$-[ $\left.\dot{q}^{w}\right]$ "to bring forth" [GT].

1.8. Ma'a sakawe "to imitate" [Ehr. $1974 \mathrm{MS}, 41]$ | WRift *îka km-/*îamk- [GT]: Irq. ŝam-im-is- "to resemble", Alg. ŝanq-am-is- "to resemble" (WRift: Ehr. 1980, 209, \#14).

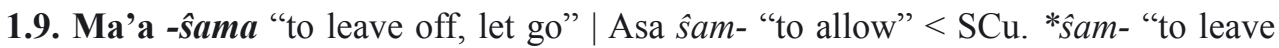
(off)" [Ehr.] (SCu.: Ehr. 1980, 208, \#5) ||| WCh.: Angas-Sura *ly ay *lay [reg. < *sam] "to lose" [GT 2004, 222] > i.a. Angas (hill) lang "to lose, be unable to find" [Flk. 1915, 233],

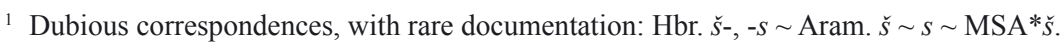


Kofyar làn (sic, -n) "to lose" [Ntg. 1967, 23], Goemay liang "to undo, unmake" [Srl. 1937, 126] < AA * $\hat{s}-m$ "to leave, lose" [GT].

1.10. Ma'a i-ŝaré “cloud” [Ehr. 1980, 214, \#6] || Bed. šāy [-y<*-r] "Wolke” [Rn. 1895, 219] || LECu.: Elmolo sếr "1. cloud, 2. rain" [Heine] ||| NOm. *šār- "cloud" [GT]: Basketo \& Wolamo šara, Kullo šariya, Male šāri | Gidicho šāra| Janjero šāru (NOm.: Bnd. 1971, \#14). Areal parallel: PKoman *šali (?) "cloud" [Bnd. 1983, 268, \#14]. Lit. for the AA etymology: Blz. 1992, 138.

1.11. Ma'a ŝ́wi "to pray, beg, ask for" [Ehr. 1974 MS, 42] | WRift *ŝa?- "to like, want" [Eld.-Mgw. 1992, 63]: Irq. \& Brg. \& Grw. \& Alg. $\hat{s} a$ ?- | ERift * $\hat{s} a$ ?- "to love, like" [GT]: Qwd. $\hat{s} a$ ?-as-, Asa $\hat{s} a$ ?-at- | Dhl. $\hat{s} a w$ - "to love, like" (SCu.: Ehr. 1980, 208; Dlg. 1973, 115) $<\mathrm{SCu} . * \hat{s} a$ ? $\sim * \hat{s} a w-$ "to love, like" [GT] || Sem. ${ }^{*} \hat{s}_{2} w$ ? [GT] $=*_{s_{2}} w y \rightarrow *^{*} \hat{s}_{2} w$ ? [Dlg.]: JAram. $s w$ ? "to wish" [Dlg.], Syr. s əwé "cupidus, studiosus" [Brk.] \| Ar. šy?: $\check{s} \bar{a}$ ? a "vouloir" [BK I 1293] = šw? [Dlg.] (Sem.: Dlg. 1987, 201; cf. Lsl. 1938, 423) ||| WCh.: Gwandara só

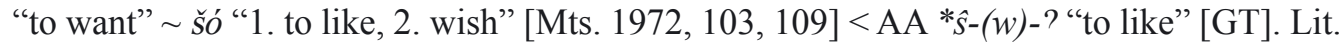
for SCu.-Ar.: Dlg. 1987, 201, \#48; HSED \#2314.

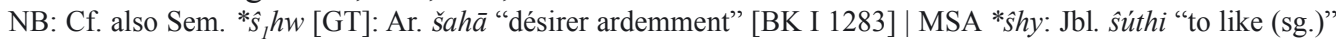
[Jns. 1981, 250].

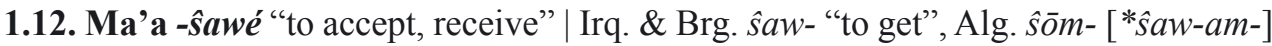

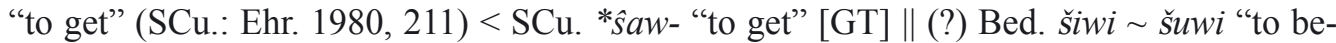
come pregnant" [Rpr. 1928, 240] ||| Eg. * šwj "to buy" [GT], preserved in the nomen agentis šwj.tj "Kaufmann" [GT: act. *"buyer"] (OK, Wb IV 434, 5-6; FD 263) || Ch. *s Ew- "to

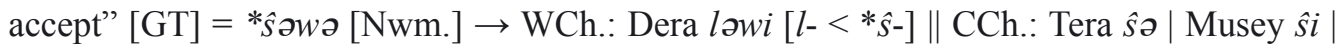
Gidar lowə [l-<*î-] (Ch.: Nwm. 1977, 22, \#1).

NB: For the semantic shift in Bed. cf. e.g. German Empfängnis "1. receipt, 2. conceiving". Cf. also Bed. šauwi šawawi šauwauwi "to get together, collect, assemble" [Rpr. 1928, 240].

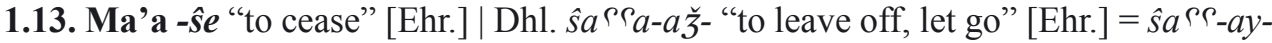
"to leave" [EEN 1989, 26] = $\hat{s} a$ ৎ- $a$ ב̌- "to leave off, abandon" [Tosco 1991, 142] (SCu.: Ehr. 1980, 209, \#15) ||| Sem.: cf. Ar. šy` II "5. reconduire qqn. et lui faire ses adieux (p.ex., un homme qui part)" [BK I 1298].

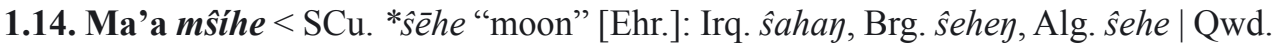
saha-yiko, Asa lehe-k (SCu.: Ehr. 1980, 212, \#30).

1.15. Ma'a ma-șom-a "Fett" [Mnh. 1906, 313] = ma-ŝ́m- $a$ "fat", $-\hat{s}$ śm- $i$ "to smear with

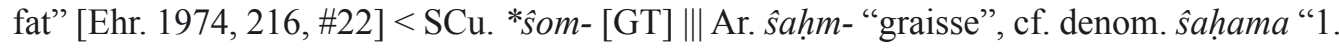
faire manger à qqn. de la graisse, 2. graisser, frotter de graisse" [BK I 1199].

NB: In Arabic we find the common Afrasian infix *- $h$ - of body parts (TAKÁcs 1997).

\section{Ma'a $\hat{s}(h l)<$ SCu. $* \hat{c}_{1}<\mathbf{A A} * \hat{c}$}

In Ma'a, the distinction between $\mathrm{SCu} .{ }^{*} \hat{s}$ and $* \hat{c}$ has not been preserved. Only the external evidence reveals that $\mathrm{SCu}$. ${ }^{*} \hat{c}$ represents the merger of two distinct Afrasian protophonemes: $\mathrm{SCu} . * \hat{c}_{1}<\mathrm{AA} * \hat{c}$, whereas SCu. $* \hat{c}_{2}<\mathrm{PAA} * \hat{c}$.

\begin{tabular}{|l|l|l|l|l|l|l|l|l|l|l|l|}
\hline WRift & Qwd. & Asa & Ma'a & Dhl. & Sem. & Hbr. & Aram. & MSA & Eg. & Ch. & AA \\
\hline$* \hat{c}_{1}$ & $\hat{c}_{1}=t l$ & $d$ & $\hat{s}=h l$ & $\hat{c}_{1}=t l$ & ${ }^{*} \hat{s}_{1}$ & $\hat{s}$ & $s$ & $* \hat{s}$ & $\grave{s}$ & $* \hat{c}$ & $* \hat{c}$ \\
\hline
\end{tabular}


2.1. Ma'a ŝá "to open"| Brg. ĉa?-as- "to uncover" (SCu.: Ehr. 1980, 215, \#12) ||| WCh.: for possible cognates see Ma'a $\hat{s} O \hat{s}$-oš $i$ "to begin" (below).

2.2. Ma'a -ŝ̀ "to rise (of sun)" [Ehr. $1974 \mathrm{MS}, 42] \mid$ (?) Brg. $\hat{c} a{ }^{\Upsilon}$-as- "to jump, fly"

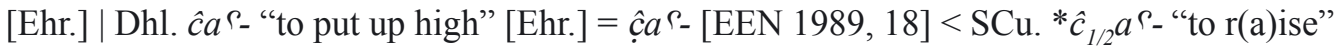
[GT].

2.3. Ma'a -ŝerú "to grow up" [Ehr.] | WRift * $\hat{c} \bar{e}[r]$ - "long, tall" [GT]: Irq. \& Grw. ĉēr "long" [Wtl.] = Irq. $\hat{c} e r$ "long, tall", $\hat{c} e r-u w$ - "to lengthen, become tall" [Ehr.], Brg. $\hat{c} \bar{e} d u$ [-d-<*-r-?] "long" [Wtl.] = ĉedi [Ehr.], Alg. $\hat{c} \bar{e} r$ "long" [Wtl.] = cer [Ehr.] (SCu.: Ehr. 1980, 216, \#17-18) < SCu. * $\hat{c}_{1} e[r]-[\mathrm{GT}]|| \mid$ Sem. * $\hat{s}_{[1]} r r$ [GT]: Geez śarara "to be high, tall" [Lsl. 1987, 534] ||| Bed. serār "lang, hochragend sein" [Rn. 1895, 205] || NAgaw: Bilin š̄̌r- "to be long, tall, far" [Rn.] = šìr- "to be long" [Bnd.], Qwara čêr- "to be far, distant" [Rn.] (NAgaw-SCu.: Dlg. 1973, 126) ||| WCh. *ŜVr- "long” [GT]: Sha šoho $\left[h<*^{*}\right]$ "wachsen, groß werden" [Jng. 1970, 288] | Maha soro "big" [Stl.], Tangale suri "long" [Krf.] | Polchi ŝari "long" [Krf.], Guruntum səri "long" [Stl.], Buli sì̀yər "long” [Krf.], Dira sìyàrì "long" [Krf.] (WCh.: Krf. 1981, \#291; Stl. 1987, 203). From AA * $\hat{c}-r$ "long” [GT].

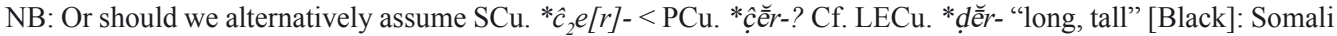
der-äd- "to become long, tall", dérer "length" | POromo *dẹer-a [Ehr.]: Oromo dếr-a "long, tall", Konso der"long, tall", d̦êr-a "tall person" etc. (ECu.: BLACK 1974, 109, 114; Dlg. 1973, 126).

2.4. Ma'a $\hat{\text { sos }} \hat{\text {-oši }}$ "to begin"| Brg. $\hat{c} a \hat{c}$-es- "to begin" (SCu.: Ehr. 1980, 214, \#5) < SCu. * $\hat{c} a \hat{c}$-as- "to begin" [GT] ||| WCh.: AS *š ̌ "to begin" [GT]: Sura $\check{s} \varepsilon \varepsilon$ "beginnen, zuerst tun" [Jng. 1963, 82], Mupun sie "to begin, start", sié (adv.) "first of all” [Frj. 1991, 55], Kofyar $\check{s}$ e "to begin" [Ntg. 1967, 35] | Dera ča "to begin” [Krf. 1981, \#416] || CCh.: Tera sàs ə or šàs ə "to begin" [Nwm. 1964, 49].

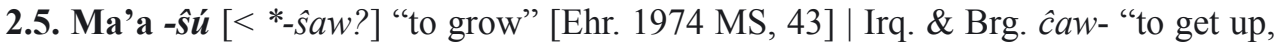
awake" (WRift: Ehr. 1980, 216) < SCu. * $\hat{c}_{1} a w$ - "to rise" [Ehr.] ||| Eg. šwj "sich erheben" (PT, Wb IV 431, 14-15) $=\check{s} w$ "to ascend" (FD 263) $\| \mid$ WCh. * $\hat{s}[a] w$ - "to rise" [GT]: NBch. * $\hat{s} a w-$

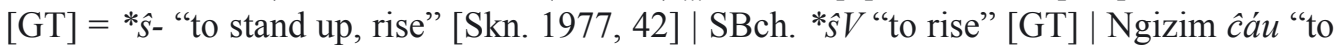
stand up, set out", ĉ̀̀̂áu "to lift, raise, cause to rise, fly up" [SchUн 1981, 162-163] (WCh.: Stl. 1987, 201) || CCh.: Logone swo "to rise" [Prh. 1972, 59] < AA * caw- "to rise" [GT].

2.6. Ma'a -ŝ́ú $\left[<*_{-} \hat{s} u k\right]$ "to fall", -šúku "to drop"|Asa $d u$ ?-um- "to fall" $<\mathrm{SCu}$. ${ }^{*} \hat{c}_{1 / 2} u k-$ “to fall” (SCu.: Ehr. 1980, 217, \#25).

\section{Ma'a $\hat{s}<\mathrm{SCu} . * \hat{c}_{2}<\mathbf{A A} * \hat{c}$}

No inner distinction is made between the derivatives of AA * $\hat{c}$ vs. AA * $\hat{c}$ in South Cushitic. This is only visible from the external cognates.

\begin{tabular}{|l|l|l|l|l|l|l|l|l|l|l|}
\hline WRift & Qwd. & Asa & Ma'a & Dhl. & Sem. & Eg. & Bed. & ECu. & Ch. & AA \\
\hline${ }^{*} \hat{c}_{2}$ & $\hat{c}_{2}=t l$ & $d$ & $\hat{s}=h l$ & $\hat{c}_{2}=t l$ & $* \underline{d}$ & $\underline{d}$ & $\underline{d}$ & $* d$ & $* \hat{c}$ & $* \hat{c}$ \\
\hline
\end{tabular}

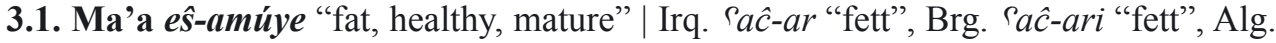

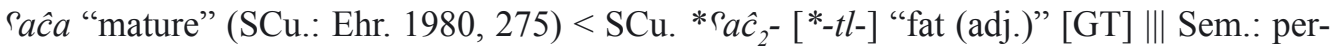
haps Ar. Pị̣d- "fort, robuste, vigoureux, infatigable, dur" [BK I 276-277] ||| Eg. ' $\underline{d}$ "Fett" $(\mathrm{OK}, \mathrm{Wb}$ I 239) < AA * 
3.2. Ma'a îsé "1. fresh, unripe, raw, 2. wet" [Ehr. 1974 MS, 28] | Irq. \& Alg. `ec "unripe, raw" (WRift: Ehr. 1980, 277, \#23) < SCu. *ৎE $\hat{c}_{2^{-}}$"unripe, raw” [GT] || LECu.: PSam *ৎayḍ

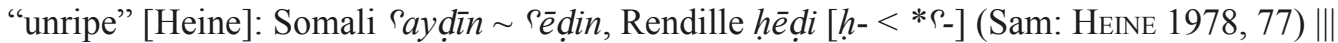
Ar. yaḍida I "être plein de vigueur, de jeunesse (se dit d'un homme, d'une plante)", II "être dans la bien-être, prospérer", yaḍd- "frais, tendre, nouveau, plein de vigueur et de santé (homme), tout jeune, né récemment (veau, etc.)" [BK II 473-474] ||| Eg. `ㅁ "wohlbehalten, unversehrt, intakt sein" (MK, Wb I 237-238, so also GHWb 165), orig. *"fresh" (?) [GT] <AA *ৎ[ay] ĉ̣- "fresh" [GT]. Lit.: Hodge 1968, 27 (Eg.-LECu.-Ar.); Dlg. 1987, 209, \#104 (LECu.-Ar.-SCu.).

\section{ABBREVIATIONS OF LANGUAGES AND RELATED TERMS}

AA: Afrasian, Afro-Asiatic (Semito-Hamitic), Akk.: Akkadian, Alg.: Alagwa, Amh.: Amharic, Ar.: Arabic, Aram.: Aramaic, AS: Angas-Sura, BA(ram.): Biblical Aramaic, Bch.: Bauchi, BD: Book of the Dead, Bed.: Bed'awye (Beja), BM: Bura-Margi, Brb.: Berber, Brg.: Burunge, BT: Bole-Tangale, Ch.: Chadic, Cpt.: Coptic, CT: coffin texts, Cu.: Cushitic, Dem.: Demotic, Dhl.: Dahalo, E: East(ern), Eg.: Egyptian, ES: Ethio-Semitic, Eth.: Ethiopian (dynasties), GR: Greek (Ptolemaic) and Roman Period, Grg.: Gurage, Grw.: Gorowa, H: Highland, Hbr.: Hebrew, Hrs.: Harsusi, IE: Indo-European, IAram.: Imperial Aramaic, Irq.: Iraqw, JAram.: Jewish Aramaic, Jbl.: Jibbali, L: Lowland, Lit.: literary texts, LE(g.): Late Egyptian, LP: Late Period, Mag.: magical texts, Med.: medical texts, ME(g.): Middle Egyptian, Mhr.: Mehri, MK: Middle Kingdom, MSA: Modern South Arabian, N: North(ern) or New, NE(g.): New Egyptian, NK: New Kingdom, O: Old, OEg.: Old Egyptian, OK: Old Kingdom, Om.: Omotic, OSA: Old (Epigraphic) South Arabian, P: Proto-, PB: Post-Biblical, Phn.: Phoenician, PT: pyramid texts, Pun.: Punic, Qtb.: Qatabanian, Qwd.: Qwadza, rel.: religious, S: South(ern), Sab.: Sabean, Sem.: Semitic, Sqt.: Soqotri, Syr.: Syriac, Tna.: Tigrinya, Ug.: Ugaritic, W: West(ern).

\section{ABBREVIATIONS OF AUTHOR NAMES}

Abr.: Abraham, BK: Biberstein Kazimirsky, Blz.: Blažek, Bnd.: Bender, Dlg.: Dolgopolsky, Een: Ehret \& Elderkin \& Nurse, Ehr.: Ehret, Eld.: Elderkin, Flk.: Foulkes, GT: Takács, Hfm.: Hoffmann, Jng.: Jungraithmayr, Jns.: Johnstone, Krf.: Kraft, Lks.: Lukas, Lsl.: Leslau, Mgw.: Maghway, Mnh.: Meinhof, Mts.: Matsushita, Ntg.: Netting, Nwm.: Newman, Prh.: Porhomovskij, Prs.: Prasse, Rn.: Reinisch, Rpr.: Roper, Skn.: Skinner, Srl.: Sirlinger, Stl.: Stolbova.

\section{LITERATURE}

Abraham R.C. 1962. Dictionary of the Hausa Language. ${ }^{2}$ London: University of London Press.

Bender Marvin Lionel. 1971. "The Languages of Ethiopia. A New Lexicostatistic Classification and Some Problems of Diffusion.” Anthropological Linguistics 13(5), 165-288.

Bender Marvin Lionel. 1983. "Proto-Koman Phonology and Lexicon.” Afrika und Übersee 66, 259-297.

Biberstein KaZimirski A. de. 1860. Dictionnaire arabe-français. Paris: Maisonneuve \& Co. Editeurs.

Black Paul David. 1974. Lowland East Cushitic: Subgrouping and Reconstruction. Ph.D. dissertation. Yale University.

BLAŽEK Václav. 1992a. “Kartvelian Material in Nostratic Lexicon: New Etymologies II.” In: Shevoroshrin 1992: $129-148$.

BLAŽEK Václav. 1992b. "Emotions in Nostratic Lexicon.” Sborník Prací Filozofické Fakulty Brněnské Univerzity. Řada Jazykovědná 40, 135-146.

Blažex Václav. 1992c. “Who Are You, Homo Sapiens Sapiens?” Human Affairs 2(2), 138-149.

DolgOPOL'SKIJ Aron Borisovič. 1973. Sravnitel'no-istoričeskaja fonetika kušitskih jazykov. Moskva: Nauka.

Dolgopolsky Aharon. 1987. "South Cushitic Lateral Consonants as Compared to Semitic and East Cushitic." In: JungraithMAYR \& MÜlLER 1987: 195-214. 
EHRET Christopher. (around 1974). Ma'a - English Vocabulary. MS. Los Angeles, California (in Ehret 1980, 390 dated for 1966-74).

EHRET Christopher. 1980. The Historical Reconstruction of Southern Cushitic Phonology and Vocabulary. Berlin: Dietrich Reimer Verlag.

Ehret Christopher, Elderkin E.D., Nurse Derek. 1989. "Dahalo Lexis and Its Sources." Afrikanistische Arbeitspapiere $18,5-49$.

Elderkin E.D., Maghway J.B. 1992. "Some West Rift Roots." African Languages and Cultures 5(1), 43-64.

FD = FAULKNER Raymond O. 1962. A Concise Dictionary of Middle Egyptian. Oxford: Clarendon Press.

Foulkes H.D. 1915. Angass Manual. Grammar, Vocabulary. London: Kegan Paul, Trench, Trübner and Co.

GHWb = HanNIG Rainer. 1995. Grosses Handwörterbuch Ägyptisch-Deutsch (2800-950 v. Chr). Mainz: Verlag Philipp von Zabern.

HeIne Bernd. 1978. "The Sam Languages. A History of Rendille, Boni and Somali." Afroasiatic Linguistics 6(2), 23-115.

CARLeton T. 1968. "Some Afroasiatic Etymologies." Anthropological Linguistics 10(3), 19-29.

Hoffmann Carl. 1975. Towards a Comparative Phonology of the Languages of the Angas-Goemai Group. MS. University of Ibadan, faculty seminar on 19 March 1975.

HSED = Orel Vladimir Émmanuilovič, Stolbova Ol'ga Valer'evna. 1995. Hamito-Semitic Etymological Dictionary. Leiden: E. J. Brill.

Johnstone T.M. 1981. Jibbāli Lexicon. London: Oxford University Press.

JungRatTHMAYR Herrmann. 1962. Wörterbuch der Angas-Sprache. MS.

JungraIthmaYR Herrmann. 1963. "Die Sprache des Sura (Maghavul) in Nordnigerien. "Afrika und Übersee 47, 8-89, 204-220.

JungraithmaYr Herrmann. 1970. Die Ron-Sprachen. Tschadohamitische Studien in Nordnigerien. Glückstadt: Verlag J. J. Augustin.

Jungraithmayr Herrmann, MülLer W.W. (eds.). 1987. Proceedings of the Fourth International Hamito-Semitic Congress. Amsterdam: John Benjamins.

Kraft Charles H. 1981. Chadic Wordlists. I-III. Berlin: Dietrich Reimer Verlag.

LESLAU Wolf. 1938. Lexique soqotri (sudarabique moderne), avec comparaisons et explications étymologiques. Paris: Librairie C. Klincksieck.

Leslau Wolf. 1987. Comparative Dictionary of Ge eez (Classical Ethiopic). Wiesbaden: Otto Harrassowitz.

Maghway J.B. 1989. "Iraqw Vocabulary.” Afrikanistische Arbeitspapiere 18, 91-118.

Matsushita Shuji. 1972. An Outline of Gwandara Phonemics and Gwandara-English Vocabulary. Tokyo: Tokyo Press.

MeInHOF Carl. 1906. "Linguistische Studien in Ostafrika. Fortsetzung." Mitteilungen des Seminars für Orientalische Sprachen 9, 278-333.

NetTing Robert M. 1967. Kofyar Vocabulary. MS.

Newman Paul. 1964. "A Word List of Tera.” Journal of West African Languages 1/2, 33-50.

Newman Paul. 1977. "Chadic Classification and Reconstructions." Afroasiatic Linguistics 5(1), 1-42.

PoRHOMOvsкis Viktor Jakovlevič. 1972. Istoričeskij konsonantizm jazykov kotoko. Moskva: Institut Jazykoznanija Akademii Nauk SSSR.

PoRHOMOvsкiJ Viktor Jakovlevič (ed.). 1987. Afrikanskoe istoričeskoe jazykoznanie. Problemy rekonstrukcii. Moskva: Nauka.

ReInISCH Leo. 1894. "Die Beḍawye-Sprache in Nordost-Afrika." III. Sitzungsberichte der Kaiserlichen Akademie der Wissenschaften. Phil.-hist. Classe 130(7), 1-80.

Roper E.-M. 1928. Tu Bedawiz. An Elementary Handbook for the Use of Sudan Government Officials. Hertford: Stephen Austin \& Sons.

Schuн Russell G. 1981. A Dictionary of Ngizim. Berkeley, California: University of California.

Shevoroshrin V. (ed.). 1992. Nostratic, Dene-Caucasian, Austric and Amerind. Bochum: Brockmeyer.

Sirlinger Eugene. 1937. Dictionary of the Goemay Language. Jos, Nigeria: Prefecture Apostolic of Jos.

SkinNER Neil. 1977. "North Bauchi Chadic Languages: Common Roots." Afroasiatic Linguistics 4(1), 1-49.

Stolbova Ol'ga Valer'evna. 1987. "Sravnitel'no-istoričeskaja fonetika i slovar' zapadnočadskih jazykov." In: PorhOMOVSKIJ 1987: 30-268.

TAKÁCs Gábor. 1997. “The Common Afrasian Nominal Class Marker *ḥ.” Studia Etymologica Cracoviensia 2, 241-273.

TAKÁCs Gábor. 2004. Comparative Dictionary of the Angas-Sura Languages. Berlin: Dietrich Reimer Verlag. 
Tosco Mauro. 1991. A Grammatical Sketch of Dahalo. Hamburg: Helmut Buske Verlag.

$\mathrm{Wb}=$ ERMAN Adolf, GRAPOw Hermann. 1957-1971. Wörterbuch der ägyptischen Sprache. I-V. ${ }^{2}$ Berlin: Akademie-Verlag.

Allatum die 19 mensis Januarii anno 2009 